\title{
Alginate/porous silica matrices for the encapsulation of living organisms: tunable properties for biosensors, modular bioreactors, and bioremediation devices
}

DOI 10.1515/mesbi-2015-0003

Received June 5, 2015; revised July 21, 2015; accepted July 22, 2015

Abstract: The encapsulation of living cells within inorganic silica hydrogels is a promising strategy for the design of biosensors, modular bioreactors, and bioremediation devices, among other interesting applications, attracting scientific and technological interest. These hostguest multifunctional materials (HGFM) combine synergistically specific biologic functions of their guest with those of the host matrix enhancing their performance. Although inorganic immobilization hosts present several advantages over their (bio)polymer-based counterparts in terms of chemical and physical stability, the direct contact of cells with silica precursors during synthesis and the constraints imposed by the inorganic host during operating conditions have proved to influence their biological response. Recently, we proposed an alternative two-step procedure including a pre-encapsulation in biocompatible polymers such as alginates in order to confer protection to the biological guest during the inorganic and more cytotoxic synthesis. By means of this procedure, whole cultures of microorganisms remain confined in small liquid volumes generated inside the inorganic host, providing near conventional liquid culture conditions. Moreover, the fact of protecting the biological guest during the synthesis of the host, allows extending the synthesis parameters beyond biocompatible conditions, tuning the microstructure of the matrix. In turn, the microstructure (porosity at the nanoscale, radius of gyration of particles composing the structure, and fractal dimension of particle clusters) is determinant of macroscopic parameters, such as optical quality and transport properties that govern the encapsulation material's performance. Here, we review the most interesting applications of the two-step procedure, making special emphasis on the optimization of optical, transport and mechanical properties of the host as well as in the interaction with the guest during operation conditions.
Keywords: Sol-gel; whole-culture encapsulation; mesoporous; bioremediation; biosensing; bioreactors

\section{Introduction}

Sol-gel technology, mainly due to its flexibility in terms of chemical diversity and processing conditions, enables the encapsulation of whole-cells, keeping the complex biological responses of the living cell. In particular, the synthesis of inorganic materials at low-temperature and biocompatible $\mathrm{pH}$ opens up new possibilities for the design of advanced host-guest multifunctional materials (HGFM) [1].

During the last decade, many efforts have been made in order to optimize both the host properties and the biocompatibility of the synthesis process allowing the encapsulation of a wide range of cell types in different hosting matrices. These host properties and guest biological responses synergistically combine enhancing the performance of the resulting hybrid materials. In the current state of affairs, one of the highest trials to overcome is to keep intact the physiological status of entrapped cells while tuning the properties of the host matrix in order to fulfill the requirements of particular applications. Direct encapsulation procedures may affect biological functions of the entrapped cells twice over: first, by contact with silica precursors in solution causing cytotoxicity due to high bioavailability of low molecular weight species of $\mathrm{Si}(\mathrm{IV})$ and second, by chemical interactions and/or mechanical

\footnotetext{
*Corresponding Author: Mercedes Perullini: INQUIMAE-DQIAQF, Facultad de Ciencias Exactas y Naturales, Universidad de Buenos Aires. Ciudad Universitaria, Pab. II, C1428EHA, Buenos Aires, Argentina, E-mail: mercedesp@qi.fcen.uba.ar; Fax: 541145763341 Mariano Calcabrini, Matıas Jobbágy, Sara A. Bilmes: INQUIMAEDQIAQF, Facultad de Ciencias Exactas y Naturales, Universidad de Buenos Aires. Ciudad Universitaria, Pab. II, C1428EHA, Buenos Aires, Argentina
} 
constraints once the inorganic hydrogel is consolidated. Although acceptable levels of viability have been reported, different microorganisms exhibited a high level of cellular stress during and after direct encapsulation procedures [2]. This is not suitable for applications such as biosensors and bioreactors, for which the entire biosynthetic machinery is compromised. For instance, when developing a biosensor based on signals triggered by biological stresses from an external stimulus such as a toxic compound present in low concentrations in the sample, this stress baseline due to host-matrix interactions raises the detection limit of the device, decreasing its performance and/or its reliability [3, 4].

Since the development of the so-called sol-gel process [5] over the past five decades, allowing the room temperature synthesis of porous silica gels, the possibility of combining advanced materials with biology has become one of the most innovative research fields [6,7]. First reports on the sol-gel encapsulation dealt with the entrapment of a wide variety of biomolecules, ranging over proteins, enzymes, and antibodies [8]. They retain their bioactivity and remain accessible to external reagents by diffusion through the nanoporous silica without significant leakage. Going one step further, the encapsulation of the whole cells retaining their metabolic activity within a stable matrix prompted new biotechnological applications. These include bioremediation devices, biosensors, bioreactors, along with health applications such as drug delivery systems, artificial organs and many promising materials for cell therapy $[9,10]$. Taking into account the diversity of applications developed to present, this paper aims to review the most interesting applications of the twostep procedure, focusing on those that have had or might have large-scale success, leaving apart the field of biomedical applications (i.e., those suitable for biomedical implants).

\section{Efficient protection of $\mathrm{Ca}$ (II)-alginate pre-encapsulation}

One of the greatest difficulties to overcome when entrapping cells inside inorganic matrices is the high cytotoxicity of the inorganic host precursors and the limitation imposed by the need of biocompatible conditions. Many efforts have been made to get over these limitations, including the development of alcohol free routes [11], the introduction of less cytotoxic alkoxide precursors, such as $\mathrm{N}$-(3-trimetoxysilylpropil)gluconamide [12], and the use of concomitant osmoprotectors (glycerol, polyethileneglicol, glycine betaine) [13, 14], among other approaches. For instance, the addition of $10 \%$ glycerol in the formulation significantly augmented (5-fold) Escherichia coli viability after 30 days encapsulation in silica hydrogels synthesized by the aqueous route [13]. However, altering the synthesis conditions by means of a synthesis additive generates a change in the matrix microstructure which can be detrimental from the application point of view, affecting optical, mechanical and/or transport properties of the final material. Taking the example of glycerol as synthesis additive, due to the high concentration needed to generate an effective osmotic protection, a 20-30\% decrease in both surface area and pore volume was evidenced. This could be attributed to its localization in the material's mesopores [13].

A simple and promising solution recently proposed is the pre-encapsulation of the cells in organic biopolymer beads [15] to provide protection to the biological guest during the inorganic and more cytotoxic synthesis. Biopolymers such as agar, carrageenan, polyacrylamide and alginate have been widely proven effective for the encapsulation of biomolecules and cells [16, 17]. Exploiting this, and inspired on the encapsulation of animal and vegetal cells performed by Boninsenga et al. in which the silica synthesis only affected the peripheral cells [18], the preencapsulation of the cells in a calcium-alginate bead followed by the sol-gel condensation of the inorganic precursors resulted in a versatile platform for the high viability encapsulation of different biological species.

The two-step procedure can be summarized as follows: (1) cells are suspended in a fresh Na-alginate solution, (2) this suspension is then added dropwise into a $\mathrm{Ca}(\mathrm{II})$ solution to form $\mathrm{Ca}(\mathrm{II})$-alginate beads, (3) the beads are placed into a mold and (4) the sol-gel precursors are added, (5) sol-gel process gives an inorganic monolith, (6) $\mathrm{Ca}$ (II) is removed via a chelating agent, and finally, (7) appropriate culture media is allowed to diffuse through the encapsulation matrix reaching the entrapped cells that remain in millimeter-sized liquid cavities within the inorganic hydrogel (see Fig. 1).

This procedure allowed the efficient encapsulation of whole bacterial cultures (Bacillus subtilis, Escherichia coli), yeasts [15], filamentous fungi (Stereum hirstum, Trametes versicolor) $[19,20]$, undifferentiated plant cells (carrot phloem and tobacco BY2 line) [21], microalgae (Chlorella vulgaris, Pseudokirchneriella subcapitata and Chlamydomonas reinhardtii) [22] and even greater multicellular organisms, such as the metazoan Daphnia magna [23]. The alginate polymer prevents the contact of the cells with the cytotoxic synthesis precursors diminishing cellular stress and enhancing initial and long-term vi- 
(2)

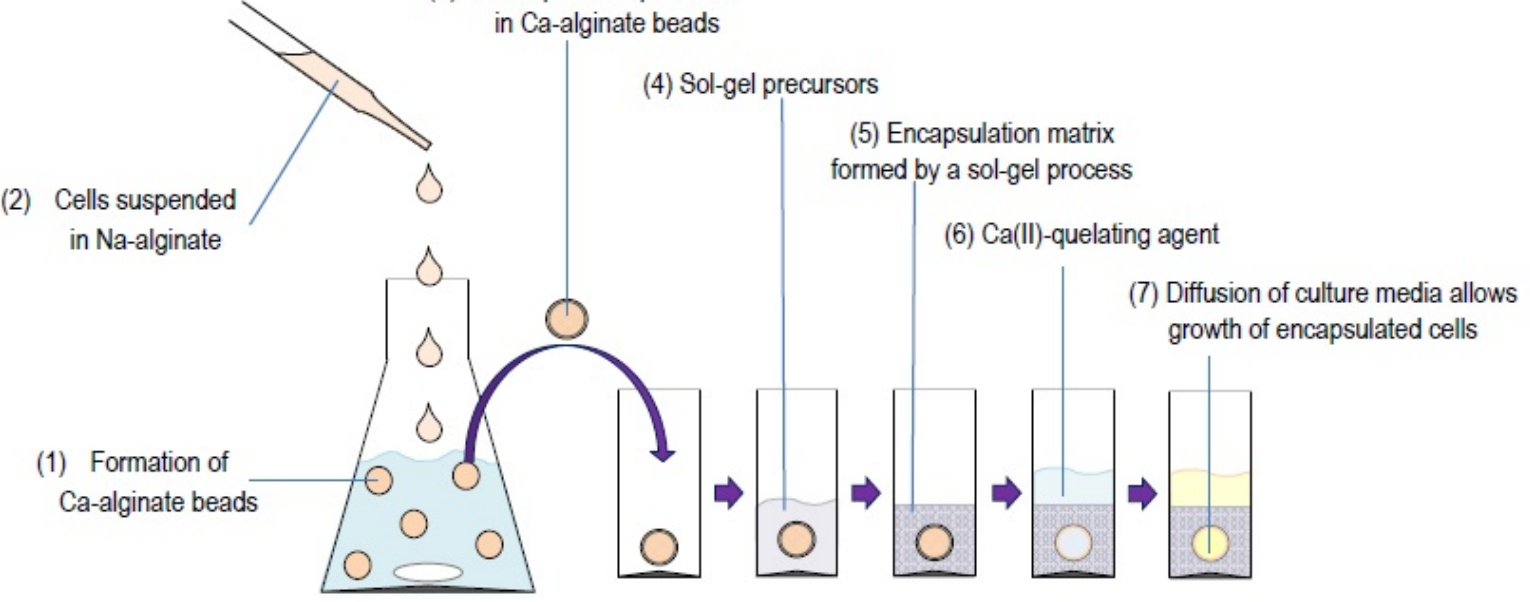

(3)

3) Cells pre-encapsulated

in Ca-alginate beads

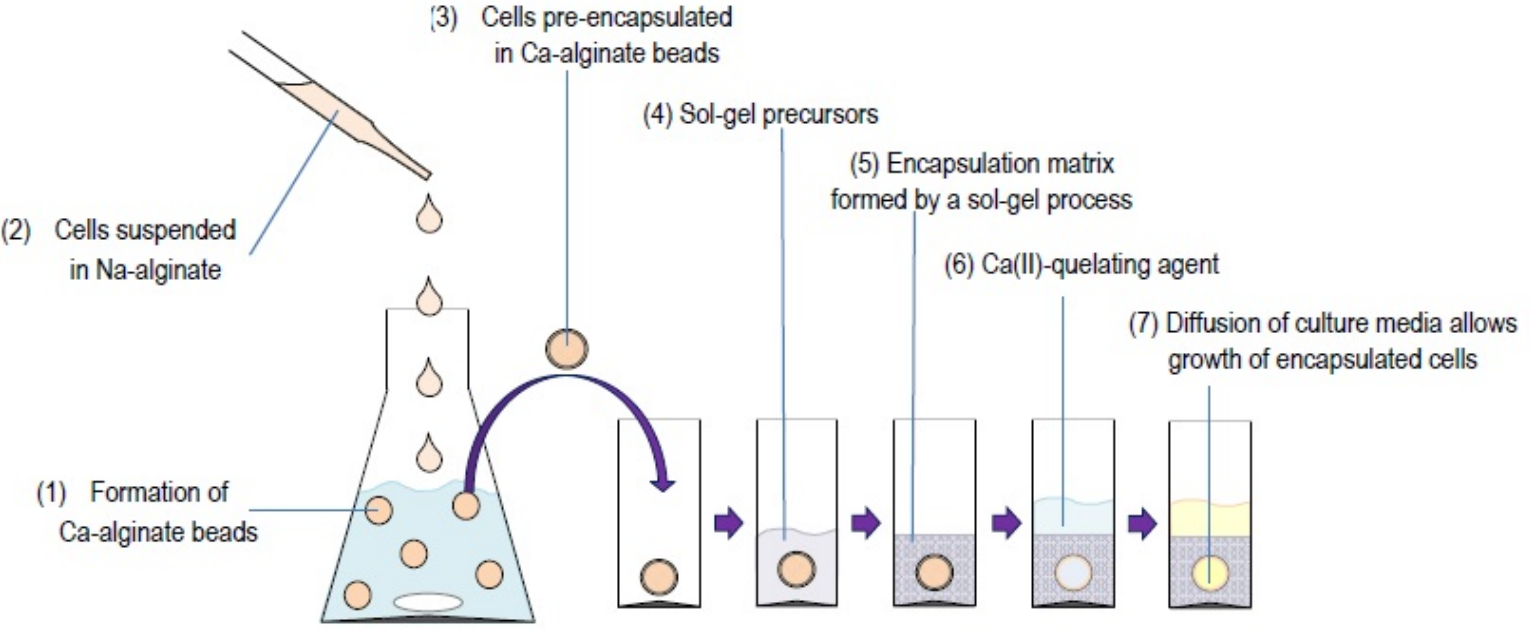

Figure 1: Schematic representation of the two-step synthesis strategy.

ability (for instance, $95 \pm 2 \%$ and $97 \pm 1 \%$ initial viability for sensitive tobacco BY2 cells and carrot tissue, respectively, as well as a high viability during the 6 months operation of the bioreactor modules) [21], which is essential for bioreactor applications. To the best of our knowledge, direct encapsulation of these plant cells within inorganic monolithic matrices has not yet been achieved. Furthermore, the generation of macrocavities inside the encapsulation matrix allows cell proliferation. This last issue is relevant for applications that require high cell density such as bioreactors and bioremediation devices. Initial works demonstrated that exponential growth during 4 days (indistinguishable from conventional liquid-cultured control) was evidenced for the encapsulation of Bacillus subtilis [15]. On the other hand, plant cells are significantly slower growing than most microbial organisms, with doubling times ranging from 20 to $100 \mathrm{~h}$ [24]. For encapsulated carrot and tobacco plant cells a doubling time of $250 \mathrm{~h}$ was obtained. Though being slower than in liquid culture media, cell growth within the inorganic material was established, which is of particular importance when the synthesis of secondary metabolites is intended.

It is worth mentioning that a recent work by Eleftheriou et al. achieved the entrapment of bacterial cells in lowconcentration silica matrices (total $\mathrm{SiO}_{2}$ content $\approx 1 \%$ ), retaining cellular division and promoter regulation, unlike almost all studies to date that have shown that these entrapped cells are unable to divide, and thus, should be expected to have reduced promoter activity [25]. On the other hand, low concentration of silica results in poor mechanical strength, not suitable for many technological applications. Although the authors do not analyze the mechani- cal properties of these matrices, similar hydrogels showed a decreasing Young modulus when lowering the $\mathrm{SiO}_{2}$ concentration $(35 \pm 3 \mathrm{MPa}, 22 \pm 3 \mathrm{MPa}$ and $18 \pm 2 \mathrm{MPa}$ for hydrogels with $16 \%, 13 \%$ and $7 \%$ of $\mathrm{SiO}_{2}$, respectively) [26].

High biocompatibility, understood not only in terms of viability but in a more comprehensive basis concerning cellular metabolism and stress, is not easy to attain. This is particularly difficult to reach, especially when dealing with eukaryotic cells, more sensitive than widely encapsulated bacteria [27]. An important reduction of the cellular stress with the model organism Saccharomyces cerevisiae was documented for two-step encapsulations compared to the direct procedure using the same inorganic synthesis (for $12.7 \% \mathrm{SiO}_{2}$ following the aqueous route, 3 -fold and 5 -fold reduction in cellular stress was evidenced for preencapsulation in $0.5 \%$ and $1.0 \% \mathrm{Ca}(\mathrm{II})$-alginate, respectively) [28].

Unlike silica coatings of alginate beads, obtained by sol-gel dip-coating [29] and gas-phase Biosil process [18], the two-step procedure leads to an inorganic monolith which exhibits higher mechanical strength. One of the most relevant advantages of the protection with such preencapsulation matrices is the possibility to use more cytotoxic synthesis conditions. This was evidenced with the successful encapsulation of $E$. coli in boehmite and zirconium oxyhydroxide hosts [30], which proved for the first time that bacterial growth within non-silica matrices was possible. In the case of the zirconia hydro gels, that had been previously proven unsuitable for cell encapsulation [31], the pre-encapsulation procedure enabled the protection of the cells from positive $\mathrm{Zr}$ (IV) oligomers and nanoparticles. This was performed by means of the addi- 
tion of a phosphate buffer to the cell suspension, previous to the alginate crosslinking, leading to the formation of a calcium phosphate precipitate that reduces the bioavailability of the cytotoxic $\mathrm{Zr}(\mathrm{IV})$ soluble species.

Another advantage of the two-step procedure is the possibility of modifying the host matrix itself by using synthesis additives that could be toxic in direct contact with entrapped cells. For instance, a silica hydrogel with embedded $\mathrm{CeO}_{2}$ nanoparticles was specially designed to protect photosynthetic guests (filtering harmful UV-light and diminishing oxidative stress of entrapped $C$. vulgaris) [32]. Similarly, the electrostatic adsorption of Rhodamine B on silica preforming particles was used to develop a silica hydrogel susceptible of being sterilized by UV-irradiation, which is highly valuable for external sterilization procedures in industrial applications [33].

In addition, for some devices that depend on a high optical quality medium, like encapsulation of photosynthetic organisms or optical based biosensors, the matrix synthesis requires high precursor concentrations and $\mathrm{pH}$ values that would not be tolerated by the cells. These conditions, however, can be successfully achieved with the two-step process, where the alginate bead is reinforced with a suitable buffer and high alginate concentration in order to mitigate the cytotoxic effects of synthesis conditions [22].

\section{Porosity of silica and alginate matrices: tuning mechanical, optical and transport properties}

The versatility of the two-step procedure will once again be apparent when considering the possibility of modifying the microstructure of the host matrix itself by extending the synthesis conditions to those that result cytotoxic in direct encapsulation procedures. It is well known that the microstructure of silica sol-gel matrices is highly dependent on the $\mathrm{pH}$ of synthesis. In particular, the TEOS derived alcohol-free route (TAFR) [11], was shown to exhibit a very interesting change in its microstructure parameters with both $\mathrm{pH}$ of the condensation stage and precursors concentration [34]. In view of its high biocompatibility, an exhaustive characterization of the silica matrices obtained by the TAFR route was made. These systems showed a very close correlation between microstructure and optical and transport properties, as well as a peculiar behavior in the range of $\mathrm{pH}$ corresponding to the minimum gelation time ( $\mathrm{pH}$ 5.5-6.0), highly relevant for the encapsulation of bio-entities [35, 36]. As shown in Fig. 2, the $\mathrm{pH}$ of the condensation stage and the concentration of silica precursors influence the size of the basic particle units, the radius of gyration of primary clusters and the fractal density of the silica network. Hence, the porosity in the mesoscale is highly dependent on the synthesis conditions. For instance, the pore volume and pore radii distribution determined by $\mathrm{N}_{2}$ sorption experiments performed at $77 \mathrm{~K}$ on the corresponding xerogels, showed marked differences for hydrogels obtained following the Silicate-Ludox ${ }^{\circledR}$ Aqueous Route (SLAR) by changing the total $\mathrm{SiO}_{2}$ content or the proportion of polymeric (silicate) to particulate $\left(\operatorname{Ludox}^{\circledR}\right)$ silica precursors. For gels with the same $\mathrm{SiO}_{2}$ content (12.7\%), while all samples exhibit a defined peak for pore radii in the 8-11 nm range, varying the ratio silicate:Ludox ${ }^{\circledR}$ from 1:3 to 1:5, generates a significant increase in the fraction of pores within the $20-30 \mathrm{~nm}$ range. Regarding the pore volume, similar values of 1.6$1.8 \mathrm{~cm}^{3} \mathrm{~g}^{-1}$ where obtained for all samples [37]. Decreasing the ratio silicate:Ludox ${ }^{\circledR}$ to 1:21 in hydrogel formulations with a comparable $\mathrm{SiO}_{2}$ content (14\%), a significant reduction in the pore volume was observed $\left(0.5 \mathrm{~cm}^{3} \mathrm{~g}^{-1}\right)$, and an extra decrease was evidenced when $10 \%$ glycerol was used as synthesis additive $\left(0.3 \mathrm{~cm}^{3} \mathrm{~g}^{-1}\right)$. These systems presented smaller specific surface areas than pure Ludox ${ }^{\circledR}\left(79 \mathrm{~m}^{2} \mathrm{~g}^{-1}\right.$ and $160 \mathrm{~m}^{2} \mathrm{~g}^{-1}$ for samples with and without the addition of $10 \%$ glycerol, respectively, compared to $220 \mathrm{~m}^{2} \mathrm{~g}^{-1}$ for the commercial nanoparticles) [13]. On the other hand, increasing the proportion of polymeric to particulate silica precursors, specific surface areas higher than pure Ludox ${ }^{\circledR}$ were observed $\left(226 \mathrm{~m}^{2} \mathrm{~g}^{-1}\right.$, $250 \mathrm{~m}^{2} \mathrm{~g}^{-1}$ and $311 \mathrm{~m}^{2} \mathrm{~g}^{-1}$ for samples with $12.7 \% \mathrm{SiO}_{2}$ and ratio silicate:Ludox ${ }^{\circledR}$ 1:5, 1:4 and 1:3, respectively). Based on these data, it can be suggested that when a low concentration of sodium silicate is used, silicates condense at the surface of pre-formed colloids which behave as nucleation sites for condensation. Gels are formed therefore via the aggregation of particles larger than in the initial sol $(12 \mathrm{~nm})$, thus explaining the decrease in specific surface area. On the contrary, for high concentration of sodium silicate, homogeneous nucleation also occurs generating smaller nanoparticles, thus increasing the specific surface area of the final material.

Optical and transport properties of these matrices can be derived from their microstructure. In particular, the diffusion of cationic species (delayed by adsorption on silica surface) can result several orders of magnitude lower than in aqueous solution, and depending on the specific surface area, this parameter can also vary 1 or 2 orders of magnitude from one hydrogel sample to another. As a general trend for TAFR hydrogels, the apparent diffusion coef- 

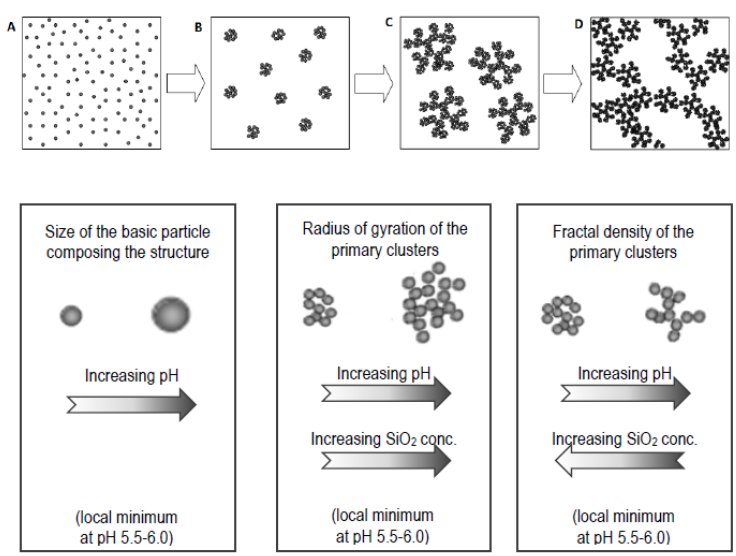

Figure 2: Above: Schematic representation of the sol-gel process: A- Hydrolyzed species can condense to form dense silica particles distributed in the sample volume. B- Particles diffuse and aggregate to form the primary clusters. C- Primary clusters collide to form higher order structures. D- Formation of the hydrogel by further interactions. Below: Microstructure parameters and their modulation by synthesis conditions.

ficient $\left(D_{a p p}\right)$ of the cationic dye crystal violet showed a 20 -fold increase when the total $\mathrm{SiO}_{2}$ content was lowered from 9.0 to 3.6\% [36]. However, the dependence of $D_{a p p}$ with the $\mathrm{pH}$ of synthesis showed a local minimum at $\mathrm{pH} \sim 6$. When diffusion is strongly retarded by adsorption, $D_{a p p}$ has been shown to be proportional to the reciprocal of specific surface area (glybet). Thus, the increase in $D_{a p p}$ with silica concentration can rely on a higher specific surface area expected in samples with higher $\mathrm{SiO}_{2}$ content. The non-monotonous trend observed with regard to the $\mathrm{pH}$ of synthesis can be understood in terms of an increased specific surface area product of a higher rate of the condensation reaction (maximum rate at $\mathrm{pH} \sim 6$ ).

Moreover, it was documented that local minimum in the apparent diffusion coefficient of cationic dyes coincides with a local maximum of the attenuance in the visible region of the spectrum for samples synthesized at $\mathrm{pH}$ 5.5-6.0, which has a strong correlation with observed singularities in the microstructure's particle and cluster size [34].

Another important issue is the microstructure of the alginate pre-encapsulation matrix. Natural polymer based hydrogels play a key role in the development of advanced biomaterials, ranging from scaffolds [38] to cell loaded artificial organs, as well as biodegradable drug delivery platforms [39]. Among them, alginate based ones demonstrated wide acceptance due to their inherent biocompatibility and versatility [40, 41]. Typically, hydrogels are prepared by submitting a soluble sodium alginate solution to crosslink its chains by M(II) or M(III) complexation, giving rise to M-Alginate hydrogels. It is known that certain properties of M-Alginate hydrogels are affected by the crosslinking procedure [42]. Among cations, $\mathrm{Ca}(\mathrm{II})$ is preferred due to its intrinsic biocompatibility. However, transition metal ones and trivalent rare earths can easily drive the process [43-47].

As an alternative procedure, the crosslinking of alginate polymer at low $\mathrm{pH}$, generates alginic acid hydrogels that reach a more stable structure, less dependent on the preparation method [48]. One particular procedure based on the in situ homogeneous release of protons resulting from the hydrolysis of D-glucono- $\delta$-lactone allows a gradient free gelation from the soluble $\mathrm{Na}(\mathrm{I})$ from to the insoluble $\mathrm{H}(\mathrm{I})$ one. The $\mathrm{pH}$ decreases to a mild value of acidity, inherently fixed by the alginate/alginic acid buffer at around $\mathrm{pH} 4$ (Sonego et al., personal communication).

\section{Bioremediation devices}

Many bacterial, algal and fungi species are well known for their ability to degrade harmful molecules. This property has been exploited to remediate contaminants and byproducts of various industrial processes with lowenergy and low-cost demanding treatments. Bio- augmentation treatments for hydrocarbon decontamination in soil and groundwater have been proposed and applied [49, 50] and dechlorinating MO's have been used to successfully clean up an aquifer [51]. Additionally, techniques like biostimulation and monitored natural attenuation are widely used to contain and treat contaminants [52-54]. Although a great variety of organisms are potentially useful for bioremediation, in situ applications are limited to native species as the introduction of exotic organisms represents an ecological risk, restricting possible interesting applications. In addition, high pollutant concentration may hinder the microorganism's metabolism [55], which represents a further limitation to bioremediation.

The sol-gel encapsulation of microorganisms emerged as a promising solution to these limitations [56]. By confining the living cells in an inorganic host, it is possible to extend the range of available organisms for bioremediation: the organisms within the host are both isolated from the medium -avoiding leakage of exotic speciesand protected against high contaminant concentrations by means of the transport properties of the host matrix. In this context, diverse applications have been developed in the last decade for remediation of industrial wastewaters and contaminant spills. In 2010, we proposed an assembly for bioremediation of polluted water using 
the two-step procedure. In this work, Stereum hirsutum fungi were encapsulated in alginate first and then inside a silica monolith prepared from colloidal silica and sodium silicate. The hydrogel adsorption properties were adjusted to regulate the transport of the contaminant in spite of retaining the ligninolytic enzymes. The so, prepared bioreactors showed high efficiency for degradation of the dye malachite green, and the modular design was proposed as a proof-of-concept for the bioremediation of contaminated waters [55]. In this direction, Tuttolomondo et al. [57] encapsulated the soil bacteria Pseudomonas sp. which was known for its capability of reducing azo groups. The cells were encapsulated in silica synthetized by acid catalyzed condensation of sodium silicate. These biocomposites showed maintained decolorizing activity for four experiments with different textile dyes: ramazol black, methyl orange and benzyl orange. Moreover, taking profit from the adsorbent capacity of sol-gel materials, encapsulated Burkholderia sp. showed reduction activity of $\mathrm{Cr}(\mathrm{VI})$ in highly contaminated aqueous and soil environments [58]. Ramachandran et al. [59] also studied the biosorption of heavy metals by encapsulation of the cyanobacteria Nostoc calcicola in two different matrices: silica dip-coated calcium alginate and silica gel from a silicate sol. In both cases the inorganic gels increased mechanical stability and adsorption capability of the cells, probably due to the interaction of the free silanol groups with the metals. This composites probed useful for several bioaccumulation cycles of $\mathrm{Cd}(\mathrm{II}), \mathrm{Cu}(\mathrm{II}), \mathrm{Cr}(\mathrm{III})$ and $\mathrm{Ni}(\mathrm{II})$. Recently a comprehensive study was performed by Soltman et al. [60] who studied a wide variety of metal bioaccumulating algal species in different inorganic matrices for biosorption of $\mathrm{Cr}(\mathrm{III}), \mathrm{Ni}(\mathrm{II}), \mathrm{Cu}(\mathrm{II})$ and $\mathrm{Pb}(\mathrm{II})$.

Regarding industrial waste treatment, Duarte et al. probed the efficient removal of phenolic compunds of olive oil mill wastewater [20] and organic content from a bleached kraft pulp mill effluent [61] by means of silicaalginate-fungi composites, which were prepared based on our previous development of modular bioremediation devices at a laboratory scale [55]. These treatments take advantage of the natural degrading properties of the strains Rhizopus oryzae, Pleurotus sajor caju and Trametes versicolor to mitigate industrial contaminants such as long chain organic acids, aromatic and fenolic compounds, and the effluent's color, which may be harmful for photosynthetic organisms if released directly to natural watercourses [20,61].

\section{Biosensing applications}

A considerable advance has been made in another interesting application of these encapsulation devices: biosensors. Different to traditional analytical methods that require reagents, biosensors can be regarded to as "reagentless". Actually, biosensors require reagents -enzymes, antibodies, cell components or whole cells- that are found immobilized. These components provide a recognition interface for various analytes which is close to or integrated with a transducer. The latter's function is to generate a specific and measurable signal to assess analytes concentration. In this field the sol-gel technology has also drawn much attention. Countless applications that imply proteins immobilized in inorganic matrices and organic polymers have been developed. Though, of particular interest in this review are those applications that rely on encapsulated cells. Among them, the encapsulation of genetically modified bacteria is a very promising approach to the development of biosensing devices. Copious work has been done in this area mainly with E. coli. Premkumar et al. [62] engineered $E$. coli to respond to general toxicity (HSP), genotoxicity and peroxides by fusing the Vibrio fischeri luxCDABE genes to grpE, recA and catalase promoters, respectively. The GMOs were encapsulated in a TMOS derived silica gel which resulted in a translucent encapsulation device that allowed stress assessment by measuring luciferase bioluminescence. Further work by the same research group [63] accounts for the use of other genetically modified $E$. coli strains that respond to fatty acids and oxidative stress. The HGFMs designed in these works are suitable for long term storage and can be used as disposable or multiple-use test kits.

Other works exploit algal sensibility to toxic compounds to determine and quantify them. HGFMs with algae are particularly useful for determining pesticides in water and soil due to the high toxicity of these compounds in photosynthetic pathways. Toxic compounds hinder the photosynthetic ability and cause shifts in the photosystems natural fluorescence. Nguyen-Ngoc et al. designed a continuous flow biosensor that lasted five weeks of uninterrupted use [64]. In this work, Chlorella vulgaris, a common model organism, is entrapped in a silica hydrogel synthetized by the aqueous route. The HGFM is in contact with an optical fiber to measure fluorescence changes in presence of DCMU (3-(3,4-dichlorophenyl)-1,1dimethylurea). The use of optical fibers in algae biosensors is quite extended. The algae Dictyosphaerium chlorelloides, Scenedesmus intermedius and Scenedesmus $s p$. were used in the same fashion by Peńa Vazquez et al. for 
triazine and urea based herbicides [65]. Here again, the fluorescence enhancement of the photosystem PSII was assessed by means of an optical fiber-HGFM junction. This work also explores the possibility to immobilize resistant mutants to increase selectivity.

Ferro et al. utilized the two-step encapsulation procedure for atrazine and DCMU detection in a similar way [66]. Chlorella vulgaris, Pseudokirchneriella subcapitata and Chlamydomonas reinhardtii were encapsulated in silica hydrogels and their fluorescence enhancement allowed concentration determination with very low detection limits. An important advantage of the two-step procedure is the co-encapsulation of multiple isolated cultures within a single common monolithic host. For instance, in biosensing applications the combination of different microorganisms in a microarray approach offers the possibility of sensing a broad range of substances in a complex sample and/or to assess a certain pollutant in a wider range of concentrations (see Fig. 3). A recent work explores the potential of miniaturization of this approach creating a lab-on-a-chip platform: patterned dot arrays of sodium alginate containing living microalgal cells were deposited onto glass carriers which were subsequently gelled using amino-functionalized silica sol to obtain reinforced alginate hydrogels [67]. Adding specificity to the detection, these biological systems can be entrapped in different host matrices allowing the selective transport of analytes by charge and/or molecular weight. Moreover, the possibility of having different species co-existing inside a single liquid volume can create an artificial ecosystem in a diminished scale that would allow ecotoxicity studies to be carried out in portable devices for on-line and in situ pollution level assessment $[23,68]$.

\section{Modular bioreactors}

Given that these HGFMs make an efficient use of their biological guest's physiological capabilities, a number of applications regarding bioreactors have been proposed. A bioreactor can be defined as a device used to carry out any kind of biological process. The biochemical reactions that take place in a bioreactor may be mediated by isolated enzymes, tissues, organelles or organisms that need to be removed for the product isolation. Herein lie two of the greatest benefits of encapsulating these mediators; by entrapping them, the mentioned issue is resolved and, additionally, the mediator can be reutilized, decreasing the production costs considerably. An interesting application of HGFMs is the development of microalgae biore- (a)
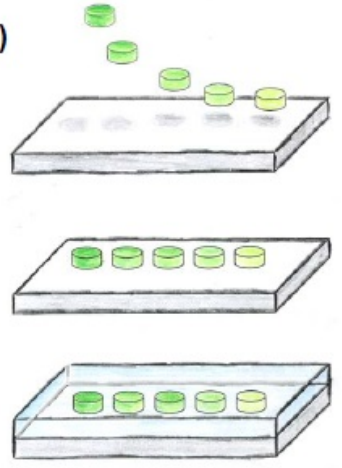

(b)
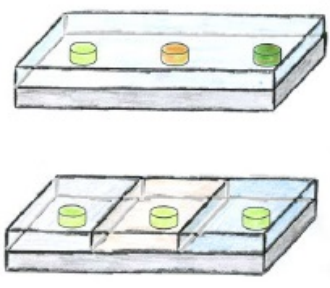

Figure 3: Scheme of synthesis of a microarray biosensing platform. (a) Sequence showing how pre-encapsulated cultures with increasing load are placed on an appropriate substrate and covered by a precursor mix, generating an inorganic hydrogel by sol-gel process. (b) Specificity can be achieved both by encapsulating alginate capsules loaded with different cellular types within a single hydrogel (upper image) or a single cellular type in each alginate capsule and by changing the properties of the inorganic host, determining for instance the transport of selective analytes (lower image).

actors for the production of nanoparticles [69, 70]. However, the advantage of the two-step procedure becomes evident when the biosynthesis of secondary metabolites is intended, as these biosynthesis products are not directly involved in the normal growth, development, or reproduction of an organism, they are synthesized at advanced stage of growth. As direct encapsulation of cells hinders the formation of cell aggregates, which induces the biosynthesis of secondary metabolites, it becomes inappropriate for such applications. On the contrary, alginate/silica hybrid platforms probed useful for the development of modular bioreactors, where carrot and tobacco plant cells were not only able to divide and grow, but also to remain viable for more than 6 months, forming vegetal calli inside a silica hydrogel [21].

\section{Conclusion}

Sol-gel chemistry allows the design of host-guest multifunctional materials with biologic activity, where the functionality is given by the fine specificity of biological functions and the material performance by the optical and mechanical properties of the host material. These macroscopic properties, as well as the necessary porosity for connecting the biological species with the external media are tuned by the synthesis parameters which determine the connectivity between units. The main advantage of inorganic matrices compared with traditional encapsulation 
in biocompatible polymers is their high chemical stability, negligible swelling, and though environmentally friendly they are not biodegradable by the embedded organisms, thus useful for long term entrapment.

The two-step encapsulation procedure improves this encapsulation since it provides a way for entrapping colonies of biological species able to grow with high viability and low stress protecting the biological guests from cytotoxic by-products produced during the inorganic synthesis. This procedure has enormously extended the range of biological species susceptible of being encapsulated inside inorganic matrices, but also permits a broad range of biological functions as microorganisms remain in nearnatural culture conditions.

This strategy opens the possibility of working in an extended range of $\mathrm{pH}$, ionic strength or precursor concentration widening the spectra of specimens to be encapsulated for a particular function in the design of bio-sensors, modular bio-reactors or bio-remediation devices.

Acknowledgement: This work has been financially supported by ANPCyT-PICT 2013-2045 and 2012-1167, CONICET GI-PIP 11220110101020 and UBACyT 20020130100048BA, from Argentina. MP, MJ and SAB are members of CONICET (Argentina).

\section{References}

[1] Blondeau, M. and T. Coradin, Living materials from sol-gel chemistry: current challenges and perspectives. Journal of Materials Chemistry, 2012. 22(42): p. 22335-22343.

[2] Kuncová, G. and J. Trögl, Physiology of microorganisms immobilized into inorganic polymers, in Handbook of Inorganic Chemistry Research. 2011. p. 53-101.

[3] Coradin, T., N. Nassif, and J. Livage, Silica-alginate composites for microencapsulation. Applied Microbiology and Biotechnology, 2003. 61(5-6): p. 429-434.

[4] Depagne, C., C. Roux, and T. Coradin, How to design cell-based biosensors using the sol-gel process. Analytical Bioanalytical Chemistry, 2011. 400(4): p. 965-976.

[5] Brinker, C.J. and G.W. Scherrer, Sol-Gel Science, 1990.

[6] Nassif, N. and J. Livage, From diatoms to silica-based biohybrids. Chemical Society Reviews, 2011. 40(2): p. 849-859.

[7] Gill, I. and A. Ballesteros, Bioencapsulation within synthetic polymers (Part 1): Sol-gel encapsulated biologicals. Trends in Biotechnology, 2000. 18(7): p. 282-296.

[8] Livage, J., T. Coradin, and C. Roux, Encapsulation of biomolecules in silica gels. Journal of Physics Condensed Matter, 2001. 13(33): p. R673-R691.

[9] Carturan, G., et al., Encapsulation of functional cells by sol-gel silica: Actual progress and perspectives for cell therapy. Journal of Materials Chemistry, 2004. 14(14): p. 2087-2098.
[10] Vallet-Regí, M. and E. Ruiz-Hernández, Bioceramics: From bone regeneration to cancer nanomedicine. Advanced Materials, 2011. 23(44): p. 5177-5218.

[11] Ferrer, M.L., F. Del Monte, and D. Levy, A novel and simple alcohol-free sol-gel route for encapsulation of labile proteins. Chemistry of Materials, 2002.14(9): p. 3619-3621.

[12] Meunier, C.F., et al., Living hybrid materials capable of energy conversion and $\mathrm{CO}_{2}$ assimilation Chemical Communications, 2010. 46: p. 3843-3859.

[13] Nassif, N., et al., A sol-gel matrix to preserve the viability of encapsulated bacteria. Journal of Materials Chemistry, 2003. 13(2): p. 203-208.

[14] Perullini, M., et al., Improving silica matrices for encapsulation of Escherichiacoli using osmoprotectors. Journal of Materials Chemistry. 21(12): p. 4546-4552.

[15] Perullini, M., et al., Cell growth at cavities created inside silica monoliths synthesized by sol-gel. Chemistry of Materials, 2005. 17(15): p. 3806-3808.

[16] De Vos, P., et al., Polymers in cell encapsulation from an enveloped cell perspective. Advanced Drug Delivery Reviews, 2014. 67-68: p. 15-34.

[17] Zimmermann, H., S.G. Shirley, and U. Zimmermann, Alginatebased encapsulation of cells: Past, present, and future. Current Diabetes Reports, 2007. 7(4): p. 314-320.

[18] Boninsegna, S., R. Dal Toso, and R. Dal Monte, Alginate microspheres loaded with animal cells and coated by a siliceous layer. Journal of Sol-Gel Science and Technology, 2003. 26(1-3): p. 1151-1157.

[19] Perullini, M., et al., Silica-alginate-fungi biocomposites for remediation of polluted water. Journal of Materials Chemistry. 20(31): p. 6479-6483.

[20] Duarte, K.R., et al., Treatment of olive oil mill wastewater by silica-alginate-fungi biocomposites. Water, Air, and Soil Pollution, 2012. 223(7): p. 4307-4318.

[21] Perullini, M., et al., Plant cell proliferation inside an inorganic host. Journal of Biotechnology, 2007. 127(3): p. 542-548.

[22] Perullini, M., et al., Sol-gel silica platforms for microalgaebased optical biosensors. Journal of Biotechnology, 2014. 179(1): p. 65-70.

[23] Perullini, M., et al., Co-encapsulation of Daphnia magna and microalgae in silica matrices, a stepping stone toward a portable microcosm. Biotechnology Reports, 2014. 4(1): p. 147-150.

[24] Kieran, P.M., P.F. MacLoughlin, and D.M. Malone, Plant cell suspension cultures: some engineering considerations. Journal of Biotechnology, 1997. 59(1-2): p. 39-52.

[25] Eleftheriou, N.M., et al., Entrapment of living bacterial cells in low-concentration silica materials preserves cell division and promoter regulation. Chemistry of Materials, 2013. 25(23): p. 4798-4805.

[26] Perullini, M., et al., Optimizing silica encapsulation of living cells: In situ evaluation of cellular stress. Chemistry of Materials, 2008. 20(9): p. 3015-3021.

[27] Kuncova, G., et al., Monitoring of the viability of cells immobilized by sol-gel process. Journal of Sol-Gel Science and Technology, 2004. 31(1-3 SPEC.ISS.): p. 335-342.

[28] Perullini, M., et al., Optimizing silica encapsulation of living cells: In situ evaluation of cellular stress. Chemistry of Materials, 2008. 20(9): p. 3015-3021.

[29] Sakai, S., et al., Newly Developed Aminopropyl-Silicate Immunoisolation Membrane for a Microcapsule-Shaped Bioarti- 
ficial Pancreas. Annals of the New York Academy of Sciences, 2001. 944(1): p. 277-283.

[30] Perullini, M., et al., Improving bacteria viability in metal oxide hosts via an alginate-based hybrid approach. Journal of Materials Chemistry, 2011. 21(12): p. 8026-8031.

[31] Amoura, M., et al., Bacteria encapsulation in colloidal inorganic matrices: is it a general method? Comptes Rendus de l'Academie des Sciences - Series Ilc: Chemistry, 2010. 13: p. 5257.

[32] Sicard, C., et al., $\mathrm{CeO}_{2}$ Nanoparticles for the Protection of Photosynthetic Organisms Immobilized in Silica Gels. Chemistry of Materials, 2011. 23(6): p. 1374-1378.

[33] Perullini, M., et al., Rhodamine $B$ doped silica encapsulation matrices for the protection of photosynthetic organisms. Journal of Biotechnology, 2014. 184: p. 94-99.

[34] Perullini, M., et al., Effect of synthesis conditions on the microstructure of TEOS derived silica hydrogels synthesized by the alcohol-free sol-gel route. Journal of Sol-Gel Science and Technology, 2011. 59(1): p. 174-180.

[35] Perullini, M., et al., New method for the simultaneous determination of diffusion and adsorption of dyes in silica hydrogels. Journal of Colloid and Interface Science, 2014. 425: p. 91-95.

[36] Perullini, M., Levinson, N., Jobbágy, M., Bilmes, S. A., Comprehensive SAXS based microstructure and transport properties characterization of biocompatible silica hydrogels, in press.

[37] Perullini, M., et al., Improving silica matrices for encapsulation of Escherichia coli using osmoprotectors. Journal of Materials Chemistry, 2011. 21(12): p. 4546-4552.

[38] Drury, J.L. and D.J. Mooney, Hydrogels for tissue engineering: scaffold design variables and applications. Biomaterials, 2003. 24(24): p. 4337-4351.

[39] Gombotz, W.R. and S.F. Wee, Protein release from alginate matrices. Advanced Drug Delivery Reviews, 1998. 31(3): p. 267-285.

[40] Smidsrod, 0. and G. Skjakbraek, Alginate as Immobilization Matrix for Cells. Trends in Biotechnology, 1990. 8(3): p. 71-78.

[41] Rowley, J.A., G. Madlambayan, and D.J. Mooney, Alginate hydrogels as synthetic extracellular matrix materials. Biomaterials, 1999. 20(1): p. 45-53.

[42] Smidsrod, 0., Properties of Poly(1,4-Hexuronates) in Gel State .2. Comparison of Gels of Different Chemical Composition. Acta Chemica Scandinavica, 1972. 26(1): p. 79-88.

[43] Brayner, R., et al., Alginate-Mediated Growth of Co, Ni, and CoNi Nanoparticles: Influence of the Biopolymer Structure. Chemistry of Materials, 2007. 19(5): p. 1190-1198.

[44] Agulhon, P., et al., Controlled synthesis from alginate gels of cobalt-manganese mixed oxide nanocrystals with peculiar magnetic properties. Catalysis Today, 2012. 189(1): p. 49-54.

[45] Monakhova, Y., et al., New mixed lanthanum- and alkaline-earth cation-containing basic catalysts obtained by an alginate route. Catalysis Today, 2012.189(1): p. 28-34.

[46] Liu, F., et al., Photoluminescent porous alginate hybrid materials containing lanthanide ions. Biomacromolecules, 2008. 9(7): p. 1945-50.

[47] Haug, A. and O. Smidsrřd, Strontium-Calcium Selectivity of Alginates. Nature, 1967. 215(5102): p. 757-757.

[48] Draget, K.I., G.S. Braek, and O. Smidsrod, Alginic Acid Gels - the Effect of Alginate Chemical-Composition and Molecular-Weight. Carbohydrate Polymers, 1994. 25(1): p. 31-38.

[49] Korda, A., et al., Petroleum hydrobcarbon bioremediation: Sampling and analytical techniques, in situ treatments and commer- cial microorganisms currently used. Applied Microbiology and Biotechnology, 1997. 48(6): p. 677-686.

[50] Farhadian, M., et al., In situ bioremediation of monoaromatic pollutants in groundwater: A review. Bioresource Technology, 2008. 99(13): p. 5296-5308.

[51] Ellis, D.E., et al., Bioaugmentation for accelerated in situ anaerobic bioremediation. Environmental Science and Technology, 2000. 34(11): p. 2254-2260.

[52] Cerqueira, V.S., et al., Comparison of bioremediation strategies for soil impacted with petrochemical oily sludge. International Biodeterioration and Biodegradation, 2014. 95(PB): p. 338-345.

[53] Sarkar, D., et al., Bioremediation of petroleum hydrocarbons in contaminated soils: Comparison of biosolids addition, carbon supplementation, and monitored natural attenuation. Environmental Pollution, 2005. 136(1): p. 187-195.

[54] Bento, F.M., et al., Comparative bioremediation of soils contaminated with diesel oil by natural attenuation, biostimulation and bioaugmentation. Bioresource Technology, 2005. 96(9): p. 1049-1055.

[55] Perullini, M., et al., Silica-alginate-fungi biocomposites for remediation of polluted water. Journal of Materials Chemistry, 2010. 20(31): p. 6479-6483.

[56] Carro, L., E. Hablot, and T. Coradin, Hybrids and biohybrids as green materials for a blue planet. Journal of Sol-Gel Science and Technology, 2014. 70(2): p. 263-271.

[57] Tuttolomondo, M.V., et al., Removal of azo dyes from water by sol-gel immobilized Pseudomonas sp. Journal of Environmental Chemical Engineering, 2014. 2(1): p. 131-136.

[58] Alvarez, G.S., et al., A functional material that combines the $\mathrm{Cr}(\mathrm{vi})$ reduction activity of Burkholderia sp. with the adsorbent capacity of sol-gel materials. Journal of Materials Chemistry, 2011. 21(17): p. 6359-6364.

[59] Ramachandran, S., et al., Nostoc calcicola immobilized in silicacoated calcium alginate and silica gel for applications in heavy metal biosorption. Silicon, 2010. 1(4): p. 215-223.

[60] Soltmann, U., Matys, S., Kieszig, G.. Pompe, W., Böttcher, H., Algae-Silica Hybrid Materials for Biosorption of Heavy Metals, J. Water Resource and Protection, 2010, 2, p. 115-122.

[61] Duarte, K.R., et al., Removal of phenolic compounds in olive mill wastewater by silica-alginate-fungi biocomposites. International Journal of Environmental Science and Technology, 2014. 11(3): p. 589-596.

[62] Premkumar, J.R., et al., Encapsulation of luminous recombinant E. coli in Sol-gel silicate films. Advanced Materials, 2001.13(23): p. 1773.

[63] Premkumar, J.R., et al., Sol-gel luminescence biosensors: Encapsulation of recombinant $E$. coli reporters in thick silicate films. Analytica Chimica Acta, 2002. 462(1): p. 11-23.

[64] Nguyen-Ngoc, H. and C. Tran-Minh, Fluorescent biosensor using whole cells in an inorganic translucent matrix. Analytica Chimica Acta, 2007. 583(1): p. 161-165.

[65] Peńa-Vázquez, E., et al., Microalgae fiber optic biosensors for herbicide monitoring using sol-gel technology. Biosensors and Bioelectronics, 2009. 24(12): p. 3538-3543.

[66] Ferro, Y., et al., Development of a biosensor for environmental monitoring based on microalgae immobilized in silica hydrogels. Sensors (Switzerland), 2012. 12(12): p. 16879-16891.

[67] Pannier, A., et al., Alginate/silica hybrid materials for immobilization of green microalgae Chlorella vulgaris for cell-based sensor arrays. Journal of Materials Chemistry B, 2014. 2(45): p. 
7896-7909.

[68] Kempf, B. and E. Bremer, Uptake and synthesis of compatible solutes as microbial stress responses to high-osmolality environments. Archives of Microbiology, 1998. 170(5): p. 319-330.

[69] Sicard, C., et al., Nano-gold biosynthesis by silica-encapsulated micro-algae: A "living" bio-hybrid material. Journal of Materials Chemistry, 2010. 20(42): p. 9342-9347.

[70] Spedalieri, C., et al., Silica@proton-alginate microreactors: A versatile platform for cell encapsulation. Journal of Materials Chemistry B, 2015. 3(16): p. 3189-3194.

\section{Bionote}

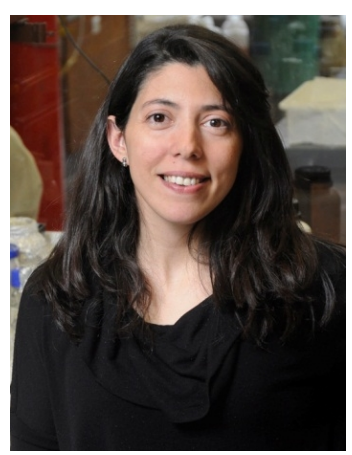

\section{Mercedes Perullini}

Mercedes Perullini is a researcher of the National Scientific and Technical Research Council of Argentina (CONICET). She graduated from Biology and received her $\mathrm{PhD}$ degree in Chemistry from Buenos Aires University. Her work at the Surfaces and Functional Materials group (INQUIMAE) is focused on the synthesis and characterization of materials with biological activity. 